\title{
Protective effects of baicalin on rabbit articular chondrocytes in vitro
}

\author{
XIANYUAN HUANG ${ }^{1,2^{*}}$, HUAYU WU ${ }^{3 *}$, LIQIN WANG $^{4}$, LI ZHENG $^{1,2}$ and JINMIN ZHAO ${ }^{1,5}$ \\ ${ }^{1}$ Guangxi Engineering Center in Biomedical Materials for Tissue and Organ Regeneration; \\ ${ }^{2}$ The Medical and Scientific Research Center; ${ }^{3}$ Department of Cell Biology and Genetics,

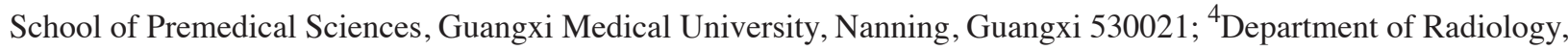 \\ The First Affiliated Hospital, Sun Yat-Sen University, Guangzhou, Guangdong 510275; ${ }^{5}$ Guangxi Key Laboratory \\ of Regenerative Medicine, Guangxi Medical University, Nanning, Guangxi 530021, P.R. China
}

Received October 16, 2015; Accepted November 4, 2016

DOI: $10.3892 /$ etm.2017.4116

\begin{abstract}
Drug therapy is one of the typical treatments for post-injury inflammation of cartilage. Traditional Chinese herbs have potential as treatments, as their long history of clinical application has demonstrated they are effective and induce minimal side effects. Baicalin is a traditional Chinese medicine that has been used to treat inflammation, fever, ulcers and cancer for hundreds of years. Previous studies have demonstrated that baicalin may decrease levels of interleukin-1 $\beta$ and suppress the expression of type-I collagen, thus attenuating cartilage degeneration. In the present study, the effect of baicalin on chondrocytes was assessed by examining the morphology, proliferation, extracellular matrix (ECM) synthesis and cartilage-specific gene expression of chondrocytes. The results indicated that baicalin may promote the proliferation of articular chondrocytes, secretion of cartilage ECM and collagen type II, aggrecan and SRY box (Sox) 9 gene upregulation. The expression of collagen I, a marker of chondrocyte dedifferentiation, was downregulated by baicalin; therefore, baicalin may maintain the phenotype of chondrocytes. Within the recommended concentrations of baicalin ranging from $0.625-6.25 \mu \mathrm{mol} / 1$ cell proliferation was increased and a $1.25 \mu \mathrm{mol} / 1$ dose of baicalin exerted the most positive effect on articular chondrocytes. The results of the present study may therefore indicate that baicalin may be used as a novel agent promoting the repair of articular cartilage damage.
\end{abstract}

Correspondence to: Professor Li Zheng or Professor Jinmin Zhao, Guangxi Engineering Center in Biomedical Materials for Tissue and Organ Regeneration, Guangxi Medical University, Science and Technology Building, 22 Shuangyong Road, Nanning, Guangxi 530021, P.R. China

E-mail: zhengli224@163.com

E-mail: zhaojinmin@126.com

*Contributed equally

Key words: baicalin, chondrocytes, joint disease, proliferation, bioactivities

\section{Introduction}

Articular cartilage has poor healing potential due to its avascularity $(1,2)$. Injured cartilage stimulates the overexpression of matrix metalloproteinases and a reduction of bioactivity in articular chondrocytes (3). The dense extracellular matrix (ECM) of cartilage hinders the migration of chondroprogenitors to the injury site, leading to irreversible cartilage loss (4).

In the repair of cartilage defects, tissue engineering strategies using carrier matrix coupled with cells to regenerate tissue are highly recommended (5). Shaped cartilage has been regenerated in vitro and in immunocompromised animals using chondrocytes and scaffolds (6). However, translation to immunocompetent animals or the clinic has proven difficult. Post-injury inflammation and sustained inflammatory reactions are the major obstacles, inhibiting sufficient ECM synthesis by chondrocytes (7). Another hurdle is the dedifferentiation of chondrocytes during the expansion in vitro. Since dedifferentiated chondrocytes produce a non-cartilage-specific ECM characterized by inferior mechanical properties, they are not suitable for cell-based therapy (8). Thus, effective anti-inflammatory mediators, which may inhibit post-traumatic cartilage inflammation and inhibit the dedifferentiation of chondrocytes to promote regeneration in cell-based therapy, are required.

There has been an increase in the utilization of herbal medicines derived from plant extracts to treat numerous clinical diseases (9). Traditional Chinese herbs have potential due to their characteristic active components, multiple targets and minimum side effects, as demonstrated by a history of clinical application (10-12). Baicalin is one of the major flavonoids isolated from the root of Scutellaria baicalensis Georgi (Huangqin in Chinese), which is used in Traditional Chinese Medicine. Baicalin has been used to treat inflammation, fever, ulcers and cancer for hundreds of years (13-18). Previous studies have demonstrated that baicalin may decrease levels of interleukin (IL)-1 $\beta$ (19) and depress the expression of collagen type I (20). As IL-1 $\beta$ is a pro-inflammatory agent and collagen type I expression is an indicator of dedifferentiation (21), baicalin may have a positive effect on inflammation and the dedifferentiation of chondrocytes. 
Based on the hypothesis that baicalin has a chondroprotective effect and improves chondrocyte healing, the present study investigated the effects of baicalin on the morphology, proliferation, cartilage-specific gene expression and ECM synthesis of chondrocytes. The present study may provide a novel clinical application for the anti-oxidant compound baicalin.

\section{Materials and methods}

Materials. Baicalin (purity, $\geq 98 \%$ ), a yellowish crystalline powder, was purchased from Chengdu Must Bio-technology Co., Ltd. (Chengdu, China). Prior to experiments, baicalin was dissolved in $2 \mathrm{ml}$ physiological saline at an initial concentration of $22.4 \mu \mathrm{mol} / \mathrm{ml}$ and stored at $-20^{\circ} \mathrm{C}$ in the dark prior its to use in subsequent experiments.

Extraction of chondrocytes. Articular cartilage cells were extracted from knee joint cartilage slices of two 5-day-old female New Zealand rabbits (weight, $80 \mathrm{~g}$ ), purchased from the Animal Experimental Center of Guangxi Medical University (Nanning, China). The two rabbits were anesthetized with $30 \mathrm{mg} / \mathrm{kg}$ 2.5\% pentobarbital sodium salt (Beijing Solarbio Science and Technology Co., Ltd., Beijing, China) and were then submerged in $75 \%$ ethanol for $5 \mathrm{~min}$. The knee joint slices were cut using ophthalmic scissors for examination in the follow-up experiments. The study was performed according to the Guide for the Care and Use of Laboratory Animals of The National Institutes of Health. The study was approved by the Committee on the Ethics of Animal Experiments of Guangxi Medical University (Nanning, China).

Articular chondrocyte culture. Articular chondrocytes were extracted from the articular cartilage slices and subsequently treated with $0.25 \%$ trypsin (Beijing Solarbio Science and Technology Co., Ltd.) in a 5 -ml centrifuge tube for $30 \mathrm{~min}$ to dissociate the epimatrix by enzymolysis. Sections were washed three times with phosphate-buffered saline (PBS; (Gibco; Thermo Fisher Scientific, Inc., Waltham, MA, USA). The perichondria of the cartilage slices were then removed for follow-up experiments. The cartilage slices were sliced into $1-\mathrm{mm}$ sections and returned to the centrifuge tube. The particles were incubated at $37^{\circ} \mathrm{C}$ with $2 \mathrm{mg} / \mathrm{ml}$ collagenase type II (Gibco; Thermo Fisher Scientific Inc.) for $4 \mathrm{~h}$. Finally, the isolated chondrocytes underwent centrifugation at room temperature at $800 \times \mathrm{g}$ RCF for $5 \mathrm{~min}$ prior to suspension in high glucose Dulbecco's modified Eagle's medium (Hyclone DMEM; GE Healthcare Life Sciences; Hyclone, Logan, UT, USA) supplemented with $10 \%(\mathrm{v} / \mathrm{v})$ fetal bovine serum (FBS; Zhejiang Tianhang Biotechnology Co., Ltd., Huzhou, China) and $1 \%(\mathrm{v} / \mathrm{v})$ penicillin and streptomycin $(100 \mathrm{U} / \mathrm{ml}$ each; Beijing Solarbio Science and Technology Co., Ltd., Beijing, China). All chondrocytes were cultured at $37^{\circ} \mathrm{C}$ in $5 \% \mathrm{CO}_{2}$ (Thermo Fisher Scientific, Inc.). The culture media of the cells were changed every $48 \mathrm{~h}$ for 7 days until $80-90 \%$ confluence was reached; the cultures were then passaged at a 1:3 ratio. Cells in the logarithmic growth phase were used for the subsequent experiments.

Cytotoxicity assay. To assess the cytotoxic effects of baicalin on articular chondrocytes, an MTT assay was performed. The cells were seeded $(2,000 /$ well $)$ in 96-well microplates in media for $24 \mathrm{~h}$. The media were then replaced with a series of baicalin concentrations $(0.625-50 \mu \mathrm{mol} / \mathrm{l})$ diluted in DMEM with $10 \%$ FBS for 2 days. Following incubation with MTT (Gibco; Thermo Fisher Scientific Inc.) at $37^{\circ} \mathrm{C}$ for $4 \mathrm{~h}$, the culture medium containing baicalin was replaced with $150 \mu 1$ dimethyl sulfoxide (Beijing Solarbio Science and Technology Co., Ltd.) in each well. The culture medium turned purple following gentle agitation for $10 \mathrm{~min}$. A microplate reader (Multiskan GO; Thermo Fisher Scientific, Inc.) was used to measure the absorbance of the solution at $570 \mathrm{~nm}$. The results of the cytotoxicity assay indicated that baicalin concentrations ranging from 0.625-6.25 $\mu \mathrm{mol} / \mathrm{l}$ promoted the growth of articular cartilage cells and the $1.25 \mu \mathrm{mol} / 1$ concentration exerted the strongest growth stimulation. Therefore, the baicalin concentrations of $0.625,1.25$ and $2.5 \mu \mathrm{mol} / 1$ were selected for further studies.

Assessment of cell proliferation and liveldead cells. Chondrocytes in high-glucose medium were seeded in 24-well plates containing cover slips, allowed to adhere for $24 \mathrm{~h}$ and incubated with baicalin at $0.625,1.25$ or $2.5 \mu \mathrm{mol} / 1$ for 2,4 or 6 days. Following three washes with PBS, cells were stained with $10 \mu \mathrm{l}$ fluorescein diacetate (FDA; Sigma-Aldrich; Merck Millipore, Darmstadt, Germany) and $5 \mu 1$ propidium iodide (PI; Sigma-Aldrich; Merck Millipore) in $1 \mathrm{ml}$ PBS in the dark for $5 \mathrm{~min}$. Subsequently, cells were observed with a fluorescent inverted phase contrast microscope (TS2R-FL; Nikon Corporation, Tokyo, Japan) and images were captured.

In another experiment, cells in high-glucose medium were seeded in 6-well plates, allowed to adhere for $24 \mathrm{~h}$ and incubated with baicalin at $0.625,1.25$ or $2.5 \mu \mathrm{mol} / 1$ for 2,4 or 6 days. Subsequently, the cells were washed three times with PBS. Following enzymolysis with $0.25 \%$ trypsin, the cells were resuspended in $1 \mathrm{ml}$ PBS containing $0.1 \mu \mathrm{g}$ proteinase $\mathrm{K}$ (BosterBio, Pleasanton, CA, USA) and incubated at $60^{\circ} \mathrm{C}$ for $6 \mathrm{~h}$. The cell suspension was stained with Hoechst 33258 (Beyotime Institute of Biotechnology, Haimen, China). Cell proliferation was assessed by measuring the absorbance value of the suspension using a Fluorescence microplate reader (FLX800; BioTek Instruments, Inc., Winooski, VT, USA) (22).

Biosynthesis ability study. Following 2, 4 and 6 days of culture in 24-well plates as described above, the cells on the cover slips were washed three times with PBS and fixed with $95 \%$ ethanol for $30 \mathrm{~min}$. The cells on the cover slips were then stained with $0.1 \%$ Safranin O (Sigma-Aldrich; Merck Millipore) for $5 \mathrm{~min}$, followed by washing with tap water for 3 min. Following sealing with resinene (Beijing Solarbio Science and Technology Co., Ltd.), the cells were imaged using an inverted phase contrast microscope (Zeiss AG, Oberkochen, Germany).

In another experiment, cells incubated with baicalin in 6-well plates for 2, 4 or 6 days as described above were washed three times with PBS and subjected to enzymolysis with $0.25 \%$ trypsin. Subsequently, cells were resuspended in $1 \mathrm{ml}$ PBS containing $0.1 \mu \mathrm{g}$ proteinase $\mathrm{K}$ at $60^{\circ} \mathrm{C}$ for $6 \mathrm{~h}$. The production of glycosaminoglycans (GAGs) was calculated by assessing the absorbance value of the cell enzyme solution with 1,9-dimethylmethylene blue using a Multiskan 
Table I. Primer sequences for reverse-transcription quantitative polymerase chain reaction experiments.

\begin{tabular}{lcc}
\hline mRNA & \multicolumn{1}{c}{ Forward primer } & \multicolumn{1}{c}{ Reverse primer } \\
\hline GAPDH & 5'-CTATAAATTGAGCCCGCAGC-3' & 5'-ACCAAATCCGTTGACTCCG-3' \\
Aggrecan & 5'-CTACACGCTACACCCTCGAC-3' & 5'-ACGTCCTCACACCAGGAAAC-3' \\
Collagen type I, $\alpha 1$ & 5'-GTTCAGCTTTGTGGACCTCCG-3' & 5'-GCAGTTCTTGGTCTCGTCAC-3' \\
Collagen type II, $\alpha 1$ & 5'-AAGCTGGTGAGAAGGGACTG-3' & 5'-GGAAACCTCGTTCACCCCTG-3' \\
Collagen type X, $\alpha 1$ & 5'-CGCTGAACGATACCAAATGCC-3' & 5'-TTCCCTACAGCTGATGGTCC-3' \\
Sox9 & 5'-AAGCTCTGGAGACTTCTGAACG-3' & 5'-CGTTCTTCACCGACTTCCTCC-3'
\end{tabular}

GAPDH, glyceraldehyde 3-phosphate dehydrogenase; Sox, SRY box 9.

GO microplate reader at $525 \mathrm{~nm}$ and comparing with the standard curve of chondroitin sulfate (23). The production of GAGs was standardized to the DNA content of the cells, which indicated the activity of cell replication in the presence of baicalin at different concentrations.

Observation of phenotype maintenance. Following 2, 4 or 6 days of culture on coverslips in 24-well plates as described above, cells were washed three times with PBS and immobilized with $95 \%$ ethanol for $30 \mathrm{~min}$. The cells were washed in PBS for $3 \mathrm{~min}$ and stained with hematoxylin and eosin (H\&E; Nanjing Jiancheng Bioengineering Institute, China). Following sealing with resinene, the cell phenotype was observed by inverted phase contrast microscopy (TS2R-FL; Nikon Corporation) and images were captured.

Secretion of collagen types I and $a$. Immunofluorescence staining for collagen type I and II was performed using collagen type I, $\alpha 1$ (COL1A1; cat. no. PB0981) and collagen type II, $\alpha 1$ (COL2A1; cat. no. BA0533) antibodies (both from Wuhan Boster Biological Technology, Ltd., Wuhan, China) according to the manufacturer's protocol. Cells on the cover slips were washed three times with PBS and fixed with $95 \%$ ethanol at room temperature for $30 \mathrm{~min}$. The cover slips were washed in PBS for 3 min and incubated with $0.01 \%$ Tritonx-100 at room temperature for $10 \mathrm{~min}$. The cells were then treated with $3 \%$ hydrogen peroxide for $10 \mathrm{~min}$ at room temperature in order to remove endogenous peroxidase activity prior to three washes with PBS for 2 min each time. The slips were then incubated with primary COL1A1 or COL2A1 antibody diluted with PBS to $1: 100$ at $37^{\circ} \mathrm{C}$ for $2 \mathrm{~h}$. Cover slips were washed three times with PBS and subsequently maintained at room temperature for $20 \mathrm{~min}$. Following three further washes with PBS, the slips were incubated with secondary antibodies (cat. no. ZDR-5306; 1:500; ZSGB-BIO, Beijing, China) at room temperature for 30 min and washed three times with PBS. The chromogenic reaction was performed using a 3,3'-diaminobenzidine tetrahydrochloride kit (Zhongshan Golden Bridge Biotechnology Co., Ltd., Beijing, China) according to the manufacturer's protocol. Following three washes in distilled water, cells were re-stained with $\mathrm{H} \& \mathrm{E}$ prior to gradual dehydration of cells with 75, 95 and $100 \%$ ethanol and mounting with resinene. The stained cells were imaged using an inverted phase contrast microscope (Nikon Corporation).
Reverse-transcription quantitative polymerase chain reaction $(R T-q P C R)$. To detect the effect of baicalin on chondrocytes at the molecular level, the expression of the chondrocyte-specific genes aggrecan, Sox9, collagen type I, collagen type II, collagen type $\mathrm{X}$ and aggrecan was analyzed by RT-qPCR. Total RNA was isolated from articular chondrocytes using the Rapture Total RNA kit (Megentec Co., Ltd., Guangzhou, China) according to the manufacturer's protocol. Complementary DNA samples were produced $(n=30)$ by the reverse transcription of RNA samples using a reverse transcription kit according to the kit instructions (K1622; Fermentas; Thermo Fisher Scientific, Inc., Pittsburg, PA, USA). SYBR-Green Master Mix (Roche Diagnostics GmbH, Mannheim, Germany) and a qPCR detection system (Realplex 4; Eppendorf, Hamburg, Germany) were used to quantify rabbit mRNA expression. The PCR primers are presented in Table I. The cDNA and primers were heated to $95^{\circ} \mathrm{C}$ to denature cDNA. Subsequent cooling to lower temperature $\left(60^{\circ} \mathrm{C}\right)$ allowed primers to hybridize to the target DNA. The reactions were repeated for 30 cycles. The $2^{-\Delta \Delta C q}$ method (24) was used to determine the gene expression relative to GAPDH.

Statistical analysis. All values are expressed as the mean \pm standard deviation. Statistical significance of multiple groups was determined through one-way analysis of variance. $\mathrm{P}<0.05$ was determined to represent a statistically significant difference using SPSS software (version 16.0; SPSS Inc., Chicago, IL, USA).

\section{Results}

Effects of baicalin on chondrocyte proliferation. Fig. 1 shows the proliferation of cells in the presence of various concentrations of baicalin relative to that of the control group. At $0.625-6.25 \mu \mathrm{mol} / \mathrm{l}$, baicalin significantly promoted chondrocyte proliferation $(\mathrm{P}<0.05)$, while it inhibited their growth at $10 \mu \mathrm{mol} / 1$. On the basis of these results, the baicalin concentrations of $0.625,1.25$ and $2.5 \mu \mathrm{mol} / 1$ were selected for further study.

Baicalin $(0.625,1.25$ and $2.5 \mu \mathrm{mol} / \mathrm{l})$ increases the proliferation and viability of chondrocytes. Chondrocytes treated with $0.625,1.25$ and $2.5 \mu \mathrm{mol} / 1$ baicalin grew faster than those in the control group and it was demonstrated that treated groups 


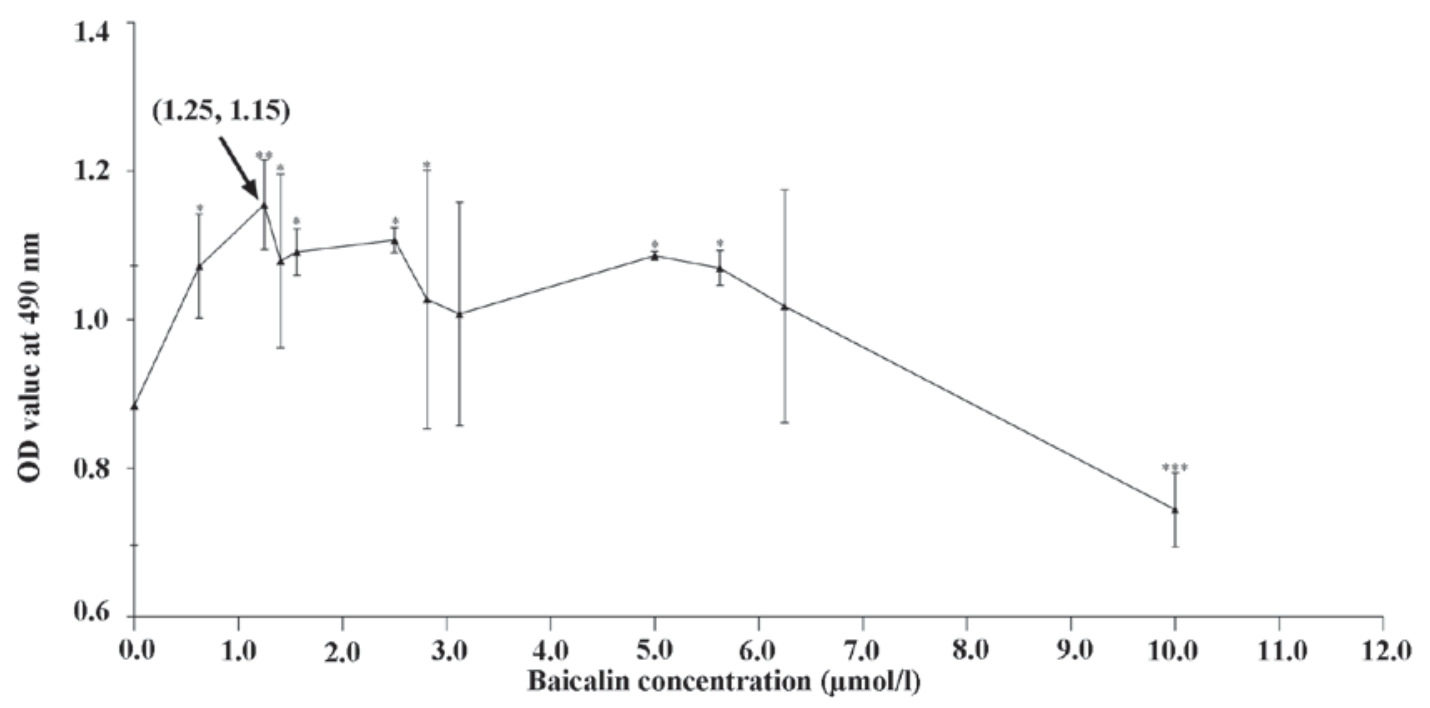

Figure 1. Cytotoxicity analysis of chondrocytes treated with different concentrations of baicalin for 2 days. The concentration at which the highest stimulation was observed $(1.25 \mu \mathrm{mol} / \mathrm{l})$ is indicated by the arrow. The coordinate $(1.25,1.15)$ is the fold change. Values are expressed as the mean \pm standard deviation $(\mathrm{n}=3) .{ }^{*} \mathrm{P}<0.05,{ }^{* *} \mathrm{P}<0.01,{ }^{* * * *} \mathrm{P}<0.001$, for the experimental group vs. the control group. OD, optical density.

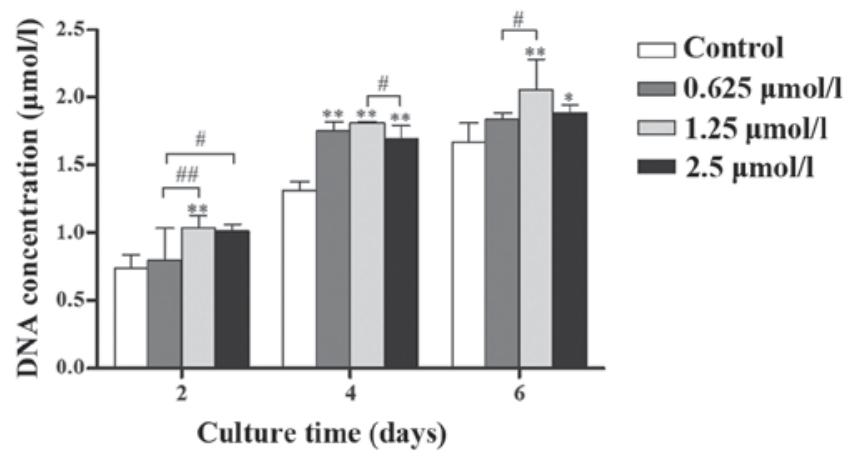

Figure 2. Quantification of cell proliferation by detection of DNA content. Chondrocytes were cultured in vitro with 0 (control), $0.625,1.25$ and $2.5 \mu \mathrm{mol} / 1$ baicalin for 2,4 and 6 days and their proliferation was assessed. Data from four independent experiments were evaluated and the mean \pm standard deviation is indicated. ${ }^{*} \mathrm{P}<0.05,{ }^{* *} \mathrm{P}<0.01$ vs. the control group. ${ }^{~} \mathrm{P}<0.05$, ${ }^{\# \#} \mathrm{P}<0.01$ between the indicated experimental groups.

had a higher DNA content (Fig. 2; $\mathrm{P}<0.05)$ at the same culture time. Among the three concentrations, $1.25 \mu \mathrm{mol} / \mathrm{l}$ baicalin most effectively promoted cell proliferation.

Live and dead cells were distinguished by FDA/PI staining. Fig. 3 demonstrates that baicalin increased the number of viable chondrocytes, which was identical to their effect on cell proliferation. Among the three baicalin groups, the concentration of $1.25 \mu \mathrm{mol} / 1$ had the largest effect.

Baicalin enhances biosynthesis in chondrocytes. GAG secretion clearly increased with time (Fig. 4) and the secretion of GAGs in baicalin-treated groups was significantly higher than that in control groups at the same culture time $(\mathrm{P}<0.05)$. Among the three baicalin groups, the $1.25 \mu \mathrm{mol} / 1$ concentration had the greatest effect on GAG secretion.

A similar result was obtained following Safranin O staining, as more chondrocyte-specific GAGs (indicated by Saffron yellow staining) were detected around the chondrocytes in the baicalin groups (Fig. 5). Among all baicalin groups, the group treated with $1.25 \mu \mathrm{mol} / 1$ exhibited the strongest staining, indicating increased GAG deposition.

Baicalin maintains the phenotype of chondrocytes. $\mathrm{H} \& \mathrm{E}$ staining was used to assess chondrocyte morphology. Fig. 6 demonstrates the morphology of articular chondrocytes following 2, 4 and 6 days in culture. The chondrocytes treated with baicalin proliferated more quickly than those in the control group. Less differentiated cells representing the typical morphology of cartilage chondrocytes were identified in the baicalin groups. Among all baicalin-treated groups, the baicalin concentration of $1.25 \mu \mathrm{mol} / 1$ exerted the strongest growth stimulatory effect on cell proliferation.

Baicalin regulates gene expression in chondrocytes. The effect of baicalin on chondrocytes was further examined at the molecular level by the analysis of gene expression. The genes assessed included collagen type I, collagen type II, collagen type X, Sox 9 and aggrecan. Following 2, 4 and 6 days culture, the expression of aggrecan, Sox 9 and collagen type II was found to be promoted by baicalin (Fig. 7). This suggested that baicalin treatment exerts auxo-action on the expression of collagen type II, Sox 9 and aggrecan and indicated that baicalin may facilitate the maintenance of the articular chondrocyte phenotype and function. By contrast, collagen type $\mathrm{X}$ was inhibited by baicalin. Thus, the present study indicated that cell hypertrophy was inhibited by baicalin. collagen type I expression was inhibited in baicalin groups on days 4 and 6 . Among all baicalin groups, the $1.25 \mu \mathrm{mol} / 1$ group exhibited the weakest expression of collagen type I and collagen type X but the strongest promotion of the cellular expression of aggrecan and collagen type II (Fig. 7).

A similar result was found following immunohistochemical staining for collagen type I (Fig. 8) and type II (Fig. 9). Positive staining was observed for cartilage-specific collagen type II in baicalin groups following 2, 4 and 6 days of culture (Fig. 9). However, less positive staining was observed for collagen type I (Fig. 8), indicating that less dedifferentiation of chondrocytes 


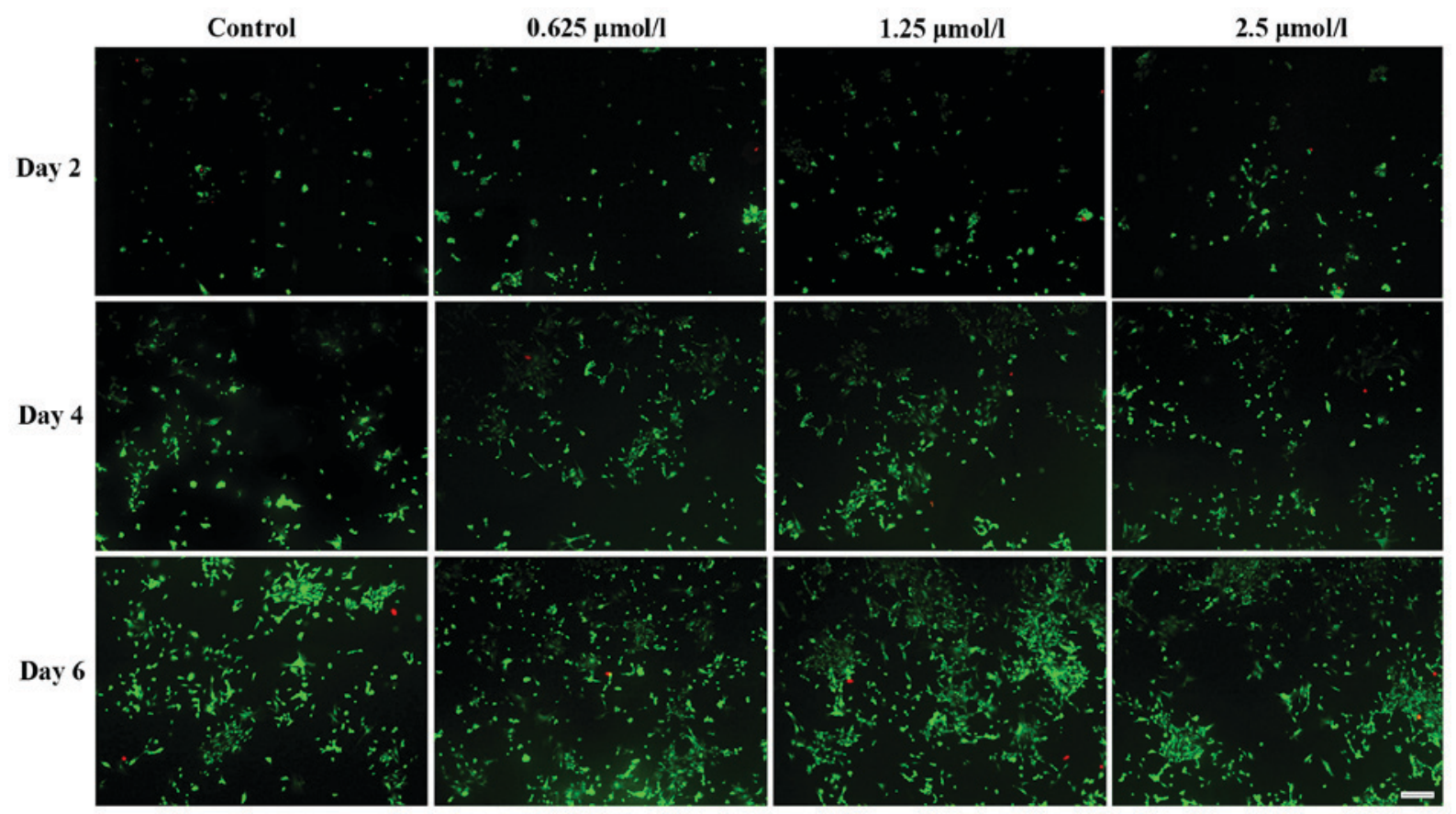

Figure 3. Fluorescein diacetate/propidium iodide staining images indicating the live (green) and dead (red) chondrocytes cultured in vitro with 0 (control), $0.625,1.25$ and $2.5 \mu \mathrm{mol} / 1$ baicalin for 2,4 and 6 days (magnification $\mathrm{x} 40$; scale bar, $200 \mu \mathrm{m}$ ).

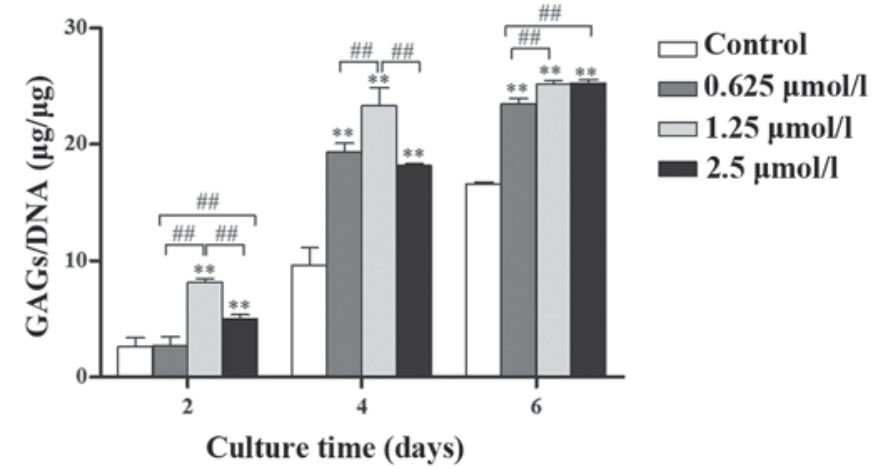

Figure 4. Quantified matrix production by GAGs $(\mu \mathrm{g})$ normalized to DNA $(\mu \mathrm{g})$. Chondrocytes were cultured in vitro with 0 (control), $0.625,1.25$ and $2.5 \mu \mathrm{mol} / 1$ baicalin for 2, 4 and 6 days. Data from four independent experiments were evaluated and the mean \pm standard deviation is indicated. ${ }^{* *} \mathrm{P}<0.01$ vs. the control group. ${ }^{\# /} \mathrm{P}<0.01$ between the indicated experimental groups.

occurred in the baicalin groups. As in the baicalin groups, more positive staining for collagen type II and less positive staining for collagen type I was observed, it was suggested that baicalin maintains the specific phenotype of articular chondrocytes.

\section{Discussion}

Articular chondrocytes possess poor self-repair ability following cartilage injury (2). In the present study, the potential application of baicalin in the treatment of arthritis was explored in improve the currently unsatisfactory clinical treatments (for example, debridement, microfracture, mosaicplasty and perichondrial grafts) available for articular diseases $(25,26)$. Baicalin is a flavonoid and an important constituent of herbs used in traditional Chinese medicine. It has been demonstrated that flavonoids have a positive effect on inflammatory diseases (27-29). In the present study, the effects of baicalin were examined by observing articular chondrocyte proliferation and phenotype maintenance. As indicated by assessing the levels of GAGs and staining with Safranin O, baicalin may promote GAG secretion in articular chondrocytes. GAGs are a series of extracellular sugar chains, which have been suggested to serve a crucial biological function in cell division (30) and may maintain the cartilage load-bearing capacity (31). In the present study, baicalin was found to upregulate the expression of collagen type II, aggrecan and Sox9, which is consistent with the increase in GAG secretion. Sox9, a chondrogenic transcription factor, serves a key role in increasing the levels of chondrogenesis (32), particularly by activating co-expression of collagen type II $(33,34)$. It has also been reported that the Sox 9 gene may upregulate aggrecan secretion $(35,36)$. These results demonstrated the crucial functions of baicalin in promoting chondrocyte proliferation and increasing cartilage matrix deposition. It was further confirmed that baicalin may influence the potential positive effect of the Sox 9 gene on articular chondrocytes, particularly that on matrix deposition.

In addition, baicalin significantly inhibited the expression of collagen type I, which is secreted by articular chondrocytes showing dedifferentiation. It has been indicated that collagen type II and cartilage-specific proteoglycan may be replaced by a complex collagen phenotype consisting of collagen type I following chondrocyte dedifferentiation (37). In the present study, RT-qPCR and immunohistochemical staining identified that, following baicalin treatment, the expression of collagen type I was decreased compared with that in the control group. In addition, collagen type $\mathrm{X}$, which is specifically identified in hypertrophic chondrocytes and during endochondral ossification (38), was clearly inhibited by baicalin. Therefore, baicalin may prevent dedifferentiation and hypertrophy of chondrocytes. 


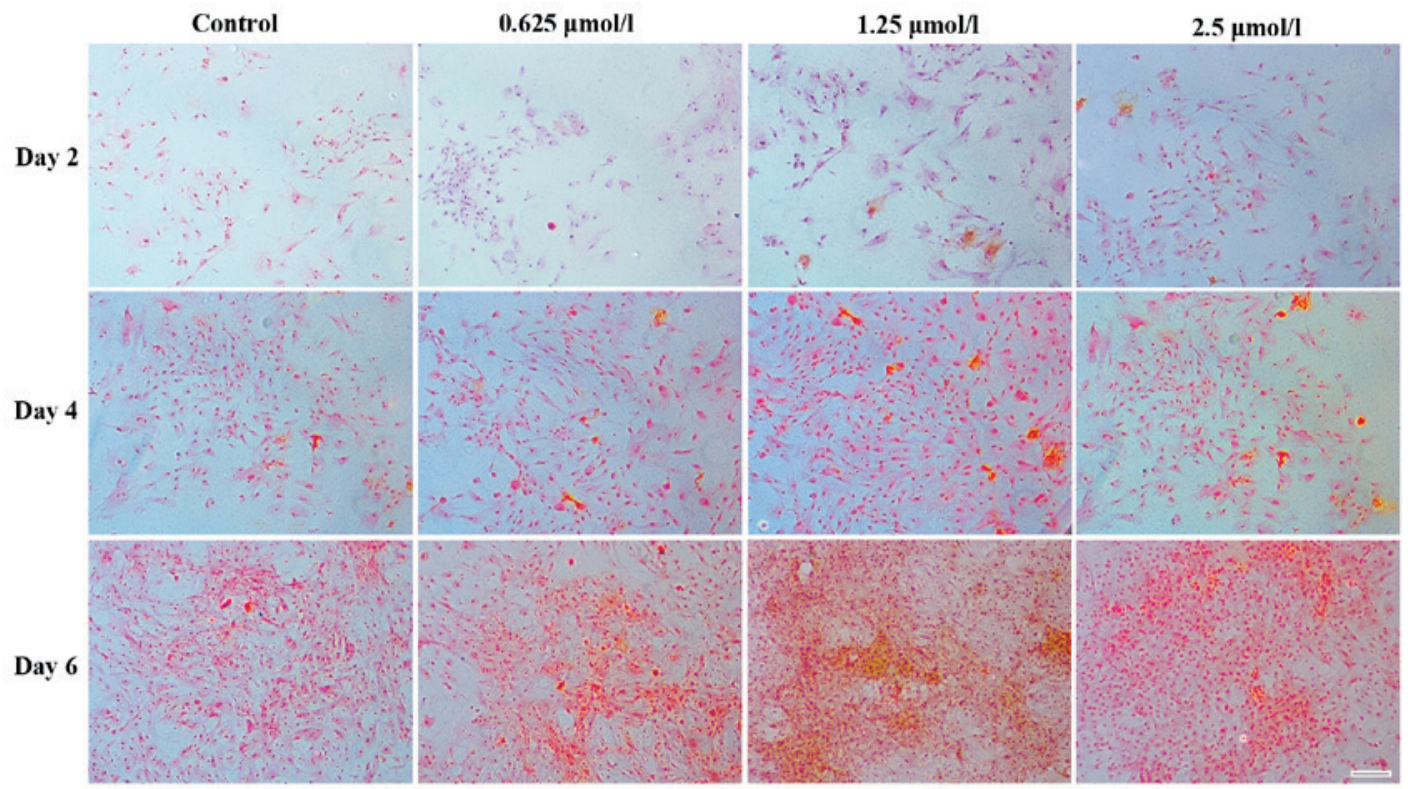

Figure 5. Safranin O staining demonstrating in vitro extracellular matrix synthesis by chondrocytes (seeding density, $2 \times 10^{4} / \mathrm{ml}$ ) cultured with 0 (control), 0.625 , 1.25 and $2.5 \mu \mathrm{mol} / 1$ baicalin for 2, 4 and 6 days (magnification x200; scale bar, $100 \mu \mathrm{m}$ ).

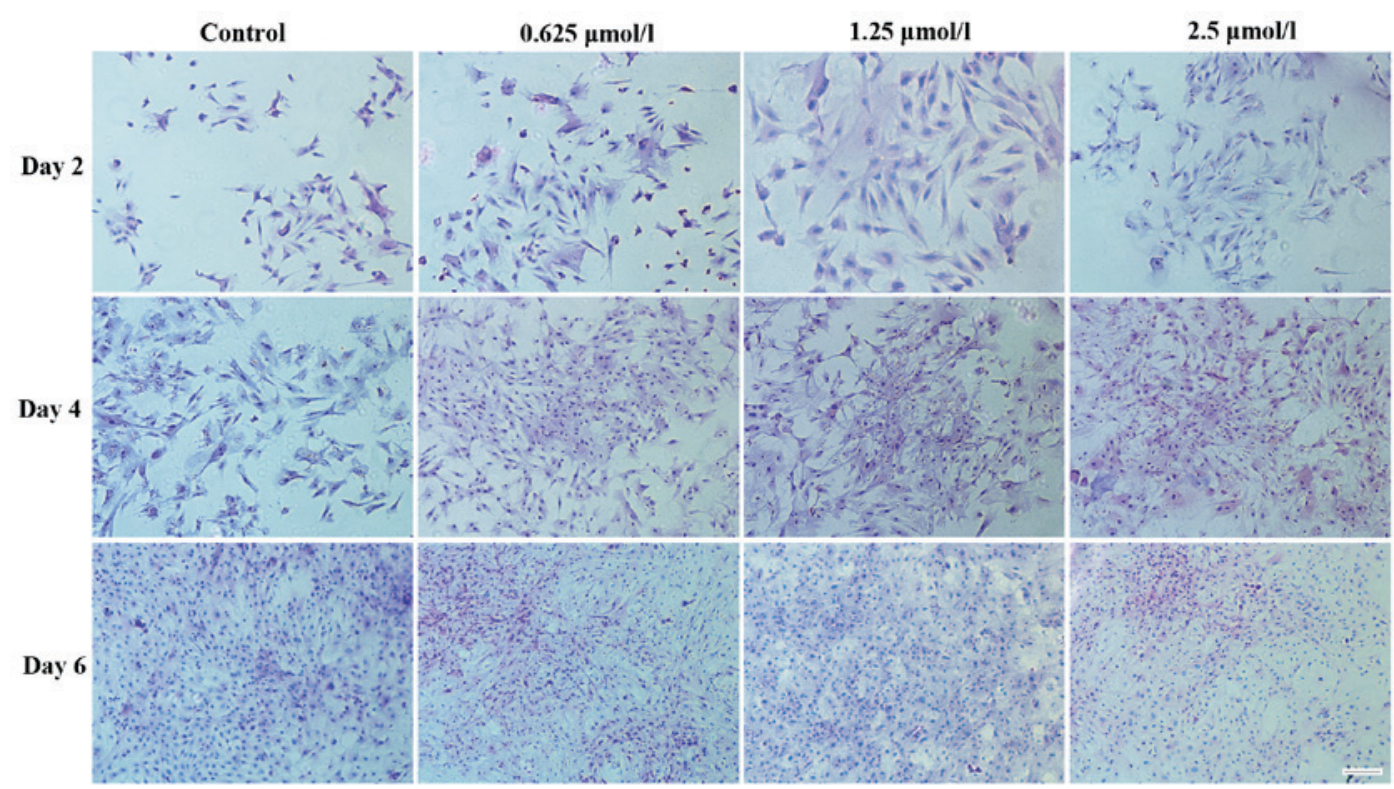

Figure 6. Hematoxylin and eosin staining images indicating the morphology of chondrocytes (seeding density, 2x104/ml) cultured in vitro with 0 (control), $0.625,1.25$ and $2.5 \mu \mathrm{mol} / 1$ baicalin for 2, 4 and 6 days. Cell (magnification x200; scale bar, $100 \mu \mathrm{m}$ ).

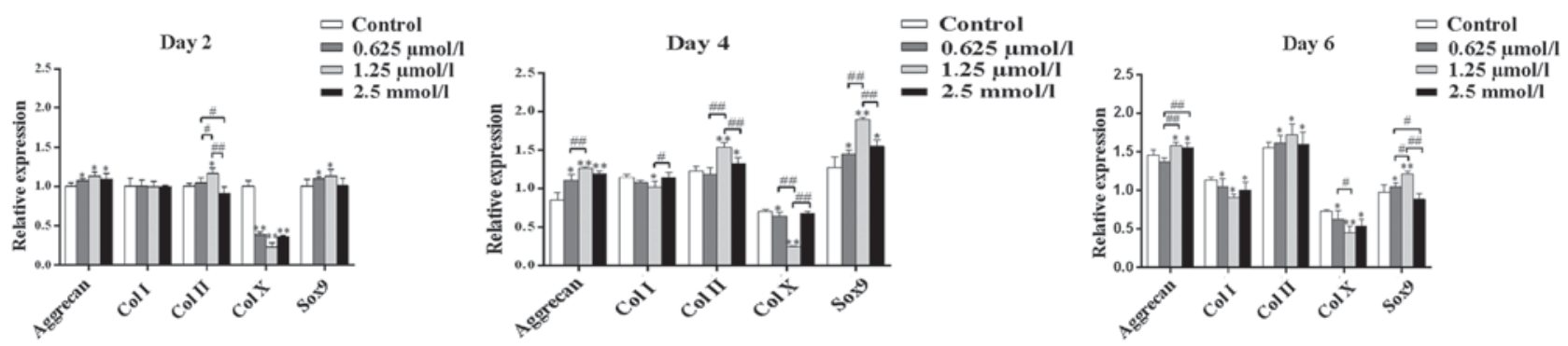

Figure 7. Quantitative comparison of extracellular matrix-associated gene expression by reverse-transcription quantitative polymerase chain reaction. The chondrocytes were cultured with 0 (control), $0.625,1.25$ and $2.5 \mu \mathrm{mol} / 1$ baicalin for 2,4 and 6 days ( $\mathrm{n}=3$ for each experiment). Levels of gene expression in baicalin media relative to the control group were analyzed using the $2^{-\Delta \Delta C q}$ method, with glyceraldehyde 3-phosphate dehydrogenase as the internal control. Values are expressed as the mean \pm standard deviation of three independent culture experiments. ${ }^{*} \mathrm{P}<0.05,{ }^{* *} \mathrm{P}<0.01$ vs. the control group. ${ }^{\sharp} \mathrm{P}<0.05$, ${ }^{\# \#} \mathrm{P}<0.01$ between the indicated experimental groups. Col, collagen; Sox, SRY box 9. 


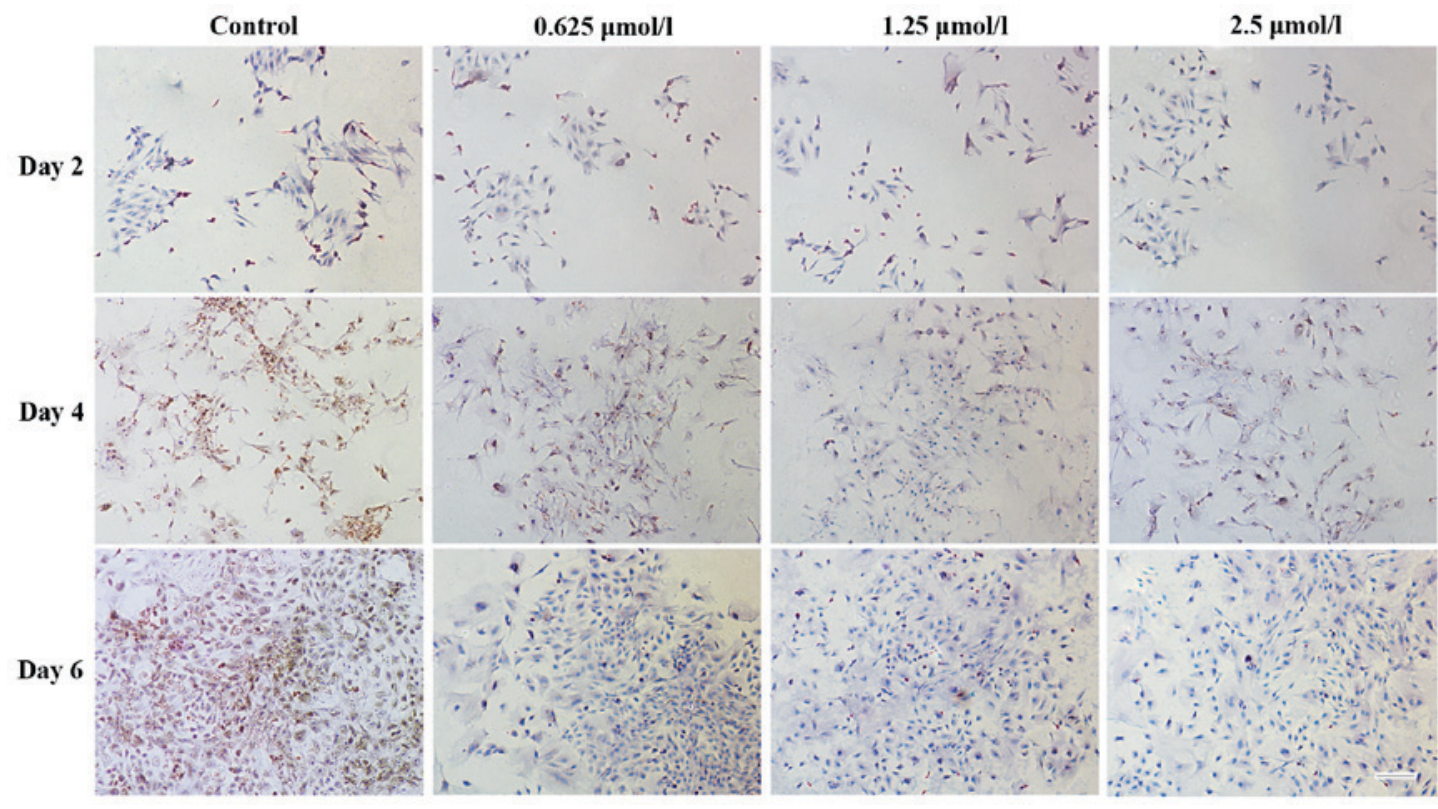

Figure 8. Immunohistochemical staining images indicating the presence of type I collagen. Chondrocytes (seeding density, 2x10 $/ \mathrm{ml}$ ) cultured in vitro with 0 (control), $0.625,1.25$ and $2.5 \mu \mathrm{mol} / 1$ baicalin for 2,4 and 6 days (magnification, $\mathrm{x} 200$; scale bar, $100 \mu \mathrm{m}$ ).

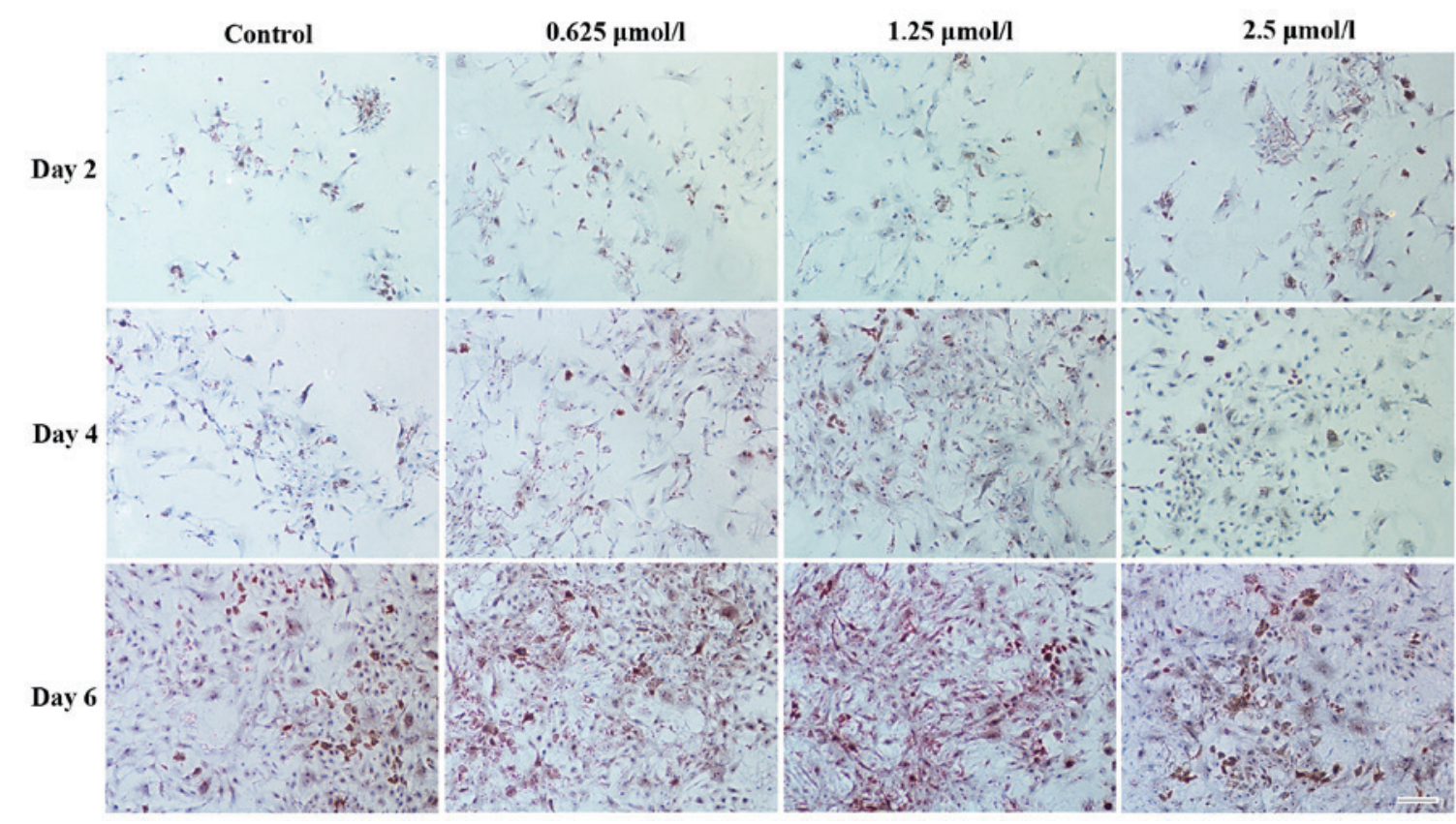

Figure 9. Immunohistochemical staining images revealing the presence of type II collagen. Chondrocytes (seeding density, 2x104/ml) cultured in vitro with 0 (control), $0.625,1.25$ and $2.5 \mu \mathrm{mol} / 1$ baicalin for 2,4 and 6 days (magnification x 200 ; scale bar, $100 \mu \mathrm{m}$ ).

Baicalin at concentrations ranging from 0.625-6.25 $\mu \mathrm{mol} / 1$ promoted the proliferation of articular chondrocytes (Fig. 1) and the DNA content was observed to increase in a dose-dependent manner. Specifically, the $1.25 \mu \mathrm{mol} / 1$ concentration of baicalin had the greatest effect in all groups, leading to the highest cell proliferation and matrix secretion.

In conclusion, the present study demonstrated that baicalin has positive effects on the proliferation and viability of rabbit articular chondrocytes. Furthermore, the results indicated the potential of baicalin to repair articular cartilage defects. Baicalin was found to promote the proliferation and maintain the phenotype of rabbit articular chondrocytes and may therefore be developed as a potential therapeutic agent to treat joint diseases caused by chondral and osteochondral lesions.

\section{Acknowledgements}

The present study was financially supported by the National Natural Science Foundation of China (grant nos. 81160221 and 81260277) and the GuangxiScientific Research and Technological Development Foundation (grant no. Guikegong 1598013-15). 


\section{References}

1. Pasold J, Zander K, Heskamp B, Gruttner C, Luthen F, Tischer T, Jonitz-Heincke A and Bader R: Positive impact of IGF-1-coupled nanoparticles on the differentiation potential of human chondrocytes cultured on collagen scaffolds. Int J Nanomedicine 10 1131-1143, 2015

2. Ishii I, Mizuta H, Sei A, Hirose J, Kudo S and Hiraki Y: Healing of full-thickness defects of the articular cartilage in rabbits using fibroblast growth factor-2 and a fibrin sealant. J Bone Joint Surg Br 89: 693-700, 2007.

3. Oda K and Minata M: Drug free remission after steroid-dependent disappearance of lymphoproliferative disorder in rheumatoid arthritis patient treated with TNF-alpha blockade: Case study. Springerplus 4: 41, 2015.

4. Buckwalter JA and Mankin HJ: Articular cartilage: Tissue design and chondrocyte-matrix interactions. Instr Course Lect 47: 477-486, 1998.

5. Abdul Rahman R, Mohamad Sukri N, Md Nazir N, Ahmad Radzi MA, Zulkifly AH, Che Ahmad A, Hashi AA, Abdul Rahman S and Sha'ban M: The potential of 3-dimensional construct engineered from poly(lactic-co-glycolic acid)/fibrin hybrid scaffold seeded with bone marrow mesenchymal stem cells for in vitro cartilage tissue engineering. Tissue Cell 47 : 420-430, 2015

6. Kojima K, Bonassar LJ, Roy AK, Mizuno H, Cortiella J and Vacanti CA: A composite tissue-engineered trachea using sheep nasal chondrocyte and epithelial cells. FASEB J 17: 823-828 2003.

7. Kojima K, Bonassar LJ, Roy AK, Vacanti CA and Cortiella J: Autologous tissue-engineered trachea with sheep nasal chondrocytes. J Thorac Cardiovasc Surg 123: 1177-1184, 2002.

8. Ting SY, Montagne K, Nishimura Y, Ushida T and Furukawa KS: Modulation of the effect of transforming growth factor- $\beta 3$ by low-intensity pulsed ultrasound on scaffold-free dedifferentiated articular bovine chondrocyte tissues. Tissue Eng Part C Methods 21: 1005-1014, 2015.

9. Liu Q, Li J, Hartstone-Rose A, Wang J, Li J, Janicki JS and Fan D: Chinese herbal compounds for the prevention and treatment of atherosclerosis: Experimental EVIDENCE AND MECHANISMS. Evid Based Complement Alternat Med 2015: $752610,2015$.

10. Lu J, Wang JS and Kong LY: Anti-inflammatory effects of Huang-Lian-Jie-Du decoction, its two fractions and four typical compounds. J Ethnopharmacol 134: 911-918, 2011.

11. Fu P, Yang L, Sun Y, Ye L, Cao Z and Tang K: Target network differences between western drugs and Chinese herbal ingredients in treating cardiovascular disease. BMC Bioinformatics 15 (Suppl 4): S3, 2014.

12. Dun RL, Yao M, Yang L, Cui XJ, Mao JM, Peng Y and Qi GC: Traditional Chinese herb combined with surgery versus surgery for varicocele infertility: A systematic review and meta-analysis. Evid Based Complement Alternat Med 2015: 689056, 2015.

13. Tsai CC, Lin MT, Wang JJ, Liao JF and Huang WT: The antipyretic effects of baicalin in lipopolysaccharide-evoked fever in rabbits. Neuropharmacology 51: 709-717, 2006.

14. He Z, Li B, Rankin GO, Rojanasakul Y and Chen YC: Selecting bioactive phenolic compounds as potential agents to inhibit proliferation and VEGF expression in human ovarian cancer cells. Oncol Lett 9: 1444-1450, 2015.

15. Xiao JR, Do CW and To CH: Potential therapeutic effects of baicalein, baicalin, and wogonin in ocular disorders. J Ocul Pharmacol Ther 30: 605-614, 2014.

16. Shen YC, Chiou WF, Chou YC and Chen CF: Mechanisms in mediating the anti-inflammatory effects of baicalin and baicalein in human leukocytes. Eur J Pharmacol 465: 171-181, 2003.

17. Ding Y, Dou J, Teng Z, Yu J, Wang T, Lu N, Wang H and Zhou C: Antiviral activity of baicalin against influenza A (H1N1/H3N2) virus in cell culture and in mice and its inhibition of neuraminidase. Arch Virol 159: 3269-3278, 2014.

18. Gao Z, Huang K and Xu H: Protective effects of flavonoids in the roots of Scutellaria baicalensis Georgi against hydrogen peroxide-induced oxidative stress in HS-SY5Y cells. Pharmacol Res 43: 173-178, 2001.

19. Chang CP, Huang WT, Cheng BC, Hsu CC and Lin MT: The flavonoid baicalin protects against cerebrovascular dysfunction and brain inflammation in experimental heatstroke. Neuropharmacology 52: 1024-1033, 2007.
20. Hu Q, Noor M, Wong YF, Hylands PJ, Simmonds MS, Xu Q, Jiang D, Hendry BM and Xu Q: In vitro anti-fibrotic activities of herbal compounds and herbs. Nephrol Dial Transplant 24: 3033-3041, 2009.

21. Zhang Y and Yao X: Role of c-Jun N-terminal kinase and p38/activation protein-1 in interleukin-1 $\beta$-mediated type I collagen synthesis in rat hepatic stellate cells. APMIS 120: 101-107, 2012.

22. Liu L, Liu Q, Lin X, Wei QJ and Zheng L: Effect of JEZTC, a synthetic compound, on proliferation and phenotype maintenance of rabbit articular chondrocytes in vitro. In Vitro Cell Dev Biol Anim 50: 982-991, 2014.

23. Farndale RW, Buttle DJ and Barrett AJ: Improved quantitation and discrimination of sulphated glycosaminoglycans by use of dimethylmethylene blue. Biochim Biophys Acta 883: 173-177, 1986.

24. Livak KJ and Schmittgen TD: Analysis of relative gene expression data using real-time quantitative PCR and the 2(-Delta Delta C (T)) Method. Methods 25: 402-408, 2001.

25. Smith GD, Knutsen G and Richardson JB: A clinical review of cartilage repair techniques. J Bone Joint Surg Br 87: 445-449, 2005.

26. Vega A, Martin-Ferrero MA, Del Canto F, Alberca M, García V, Munar A, Orozco L, Soler R, Fuertes JJ, Huguet M, et al: Treatment of knee osteoarthritis with allogeneic bone marrow mesenchymal stem cells: A randomized controlled trial. Transplantation 99: 1681-1690, 2015.

27. Lee JH, Ahn J, Kim JW, Lee SG and Kim HP: Flavonoids from the aerial parts of Houttuynia cordata attenuate lung inflammation in mice. Arch Pharm Res 38: 1304-1311, 2015.

28. Liu JJ, Huang TS, Cheng WF and Lu FJ: Baicalein and baicalin are potent inhibitors of angiogenesis: Inhibition of endothelial cell proliferation, migration and differentiation. Int J Cancer 106: 559-565, 2003.

29. Zhang D, Huang B, Xiong C and Yue Z: Pinocembrin inhibits matrix metalloproteinase expression in chondrocytes. IUBMB Life 67: 36-41, 2015

30. Mizuguchi S, Uyama T, Kitagawa H, Nomura KH, Dejima K, Gengyo-Ando K, Mitani S, Sugahara K and Nomura K: Chondroitin proteoglycans are involved in cell division of Caenorhabditis elegans. Nature 423: 443-448, 2003.

31. Ancsin JB: Amyloidogenesis: Historical and modern observations point to heparan sulfate proteoglycans as a major culprit. Amyloid 10: 67-79, 2003.

32. Shi S, Wang C, Acton AJ, Eckert GJ and Trippel SB: Role of Sox 9 in growth factor regulation of articular chondrocytes. J Cell Biochem 116: 1391-1400, 2015.

33. Davies SR, Chang LW, Patra D, Xing X, Posey K, Hecht J, Stormo GD and Sandell LJ: Computational identification and functional validation of regulatory motifs in cartilage-expressed genes. Genome Res 17: 1438-1447, 2007.

34. Li Y, Tew SR, Russell AM, Gonzalez KR, Hardingham TE and Hawkins RE: Transduction of passaged human articular chondrocytes with adenoviral, retroviral, and lentiviral vectors and the effects of enhanced expression of SOX9. Tissue Eng 10: 575-584, 2004.

35. Zamani S, Hashemibeni B, Esfandiari E, Kabiri A, Rabbani H and Abutorabi R: Assessment of TGF-b3 on production of aggrecan by human articular chondrocytes in pellet culture system. Adv Biomed Res 3: 54, 2014.

36. Svala E, Löfgren M, Sihlbom C, Rüetschi U, Lindahl A, Ekman S and Skiöldebrand E: An inflammatory equine model demonstrates dynamic changes of immune response and cartilage matrix molecule degradation in vitro. Connect Tissue Res 56: 315-325, 2015.

37. Xing R, Kong Q, Xiang Z, Yang J, Luo J, Deng L and Xie H: Preliminary study on microRNA regulated osteogenic and chondrogenic differentiation of mouse stem cells. Zhongguo Xiu Fu Chong Jian Wai Ke Za Zhi 28: 1009-1016, 2014 (In Chinese).

38. Yang X, Trehan SK, Guan Y, Sun C, Moore DC, Jayasuriya CT and Chen Q: Matrilin-3 inhibits chondrocyte hypertrophy as a bone morphogenetic protein-2 antagonist. J Biol Chem 289: 34768-34779, 2014. 